\title{
Natriuretic peptides: are these new links in the hepatorenal connections?
}

In 1981 , de Bold et $a l^{1}$ discovered that the heart produced a peptide factor, subsequently named atrial natriuretic peptide (ANP), with potent actions affecting sodium metabolism and blood pressure. Since then, other related peptides, first brain natriuretic peptide (BNP), and more recently $C$ type natriuretic peptide (CNP) were isolated. These peptides share sufficient similarities in structure, gene regulation, and metabolism to be grouped together as a family of peptides. It was soon realised that these hormones have multiple and varied actions beyond their natriuretic property, that not all are natriuretic, and they may have paracrine as well as endocrine actions. ${ }^{2}$ These diverse effects are mediated through specific membrane associated natriuretic peptide receptors (NPRs), with a receptor subtype being selective for each peptide. Despite this, they have remained together as a family of natriuretic peptides.

Atrial natriuretic peptide, the prototype of the natriuretic peptides, is secreted from the atria. ANP has potent haemodynamic and renal effects. It is natriuretic, vasodilatory as well as being an antagonist to the actions of the renin-angiotensin-aldosterone system, and therefore important in the regulation of blood pressure. ANP has numerous other endocrine actions in vitro and in experimental animals including inhibition of ACTH and arginine vasopressin secretions, as well as inhibition of thirst. More recently, an in vitro anti-proliferative effect of ANP on vascular smooth muscle cells has also been shown. ${ }^{3}$ Brain natriuretic peptide is something of a misnomer. Although originally isolated in the brain, its main site of synthesis is the heart. It binds to the same receptor and has similar biological effects as ANP.

$C$ type natriuretic peptide, the newest member of the natriuretic peptide family, was originally isolated from porcine brain. Little CNP is detected in cardiac tissues; in contrast, significant concentrations are found in the brain, vascular endothelium, and the kidney. ${ }^{4}$ The role of CNP as a circulating hormone remains unproved, because the plasma concentrations are low and there is little evidence to support that plasma values are subject to regulation. It therefore has been postulated to be a paracrine hormone. Despite its presence, together with its receptor in the kidney, it has no natriuretic action in humans. Recent work has directed towards identifying a vascular CNP system, with CNP exerting an anti-proliferative effect on vascular smooth muscle cells, ${ }^{5}$ thereby acting as a mediator of vascular remodelling.

Thus we read with interest the article by Vollmar et al (see page 145) describing the identification using reverse transcriptase polymerase chain reaction of both ANP, CNP and their respective NPRs as well as the clearance receptor, NPR-C, in liver tissue. Brain natriuretic peptide, however, was not found in the liver. Furthermore, expression of both ANP and CNP were severalfold higher in liver tumour tissue, yet only increased levels of receptor for ANP but not for CNP were found in the same tumour tissue.
The presence of natriuretic peptides and their receptors in liver tissue would suggest that they may have autocrine or paracrine functions. Plasma ANP concentrations have been demonstrated to be normal or increased in cirrhosis, ${ }^{6}$ thought to be due to increased production from atrial stretch as a result of hypervolaemia on the right side of the circulation. Systemic arterial vasodilatation is commonly seen in these patients, and this is in keeping with ANP as being one of the possible vasodilators involved. Yet these patients clearly demonstrate sodium retention. This apparent paradox would suggest that other factors are opposing, or that these patients have developed renal unresponsiveness to the natriuretic effects of ANP. A local hepatic ANP system would suggest that apart from its systemic effects, ANP may exert additional effects through its autocrine or paracrine action in the liver. The authors have indicated possible hepatic effects of ANP including protection against ischaemia and reperfusion injury, which would be consistent with its known vasodilatory effects. Although speculative, it is also possible that the hepatic ANP system is an inbuilt local vasodilatory mechanism to counteract the sinusoidal portal hypertension in cirrhosis. Sinusoidal portal hypertension is associated with renal sodium retention, because the reduction in sinusoidal portal pressure after the insertion of a transjugular intrahepatic portosystemic shunt is associated with a natriuresis. $^{7}$ This local hepatic ANP system may well provide an indirect signal to the kidney, via some hepatorenal interaction, to augment the natriuretic response. The local hepatic ANP system may also provide vasomotor control of the microcirculation of the liver, as part of the regulatory mechanism to maintain a constant hepatic circulation.

The fact that the expression of ANP and its receptor are severalfold higher in liver tumour tissue would suggest that ANP is closely linked to liver tissue growth, either as a stimulator for growth or as a response in an attempt to contain tissue growth. As the authors clearly pointed out, it is uncertain whether the increased ANP expression is of hepatocellular or endothelial origin. It is possible that ANP may be elaborated from the endothelial cells, as hepatocellular carcinomas are known to be very vascular tumours. It will be interesting to compare the gene expression of ANP in normal versus cirrhotic livers. If the ANP gene expression is increased in the latter, then its role in regeneration of liver cells can be entertained.

The presence of CNP and its receptor in liver tissue may merely represent a redundant hepatic CNP system, similar to its renal counterpart that is not associated with a natriuretic action. However, the known anti-proliferative action of CNP on vascular endothelium would suggest a definitive role of CNP in the liver, although exactly what that is has not been elucidated. The release of CNP mRNA is stimulated in cultured bovine aortic endothelial cells by the addition of $A N P,{ }^{8}$ it is therefore possible that CNP provides a vasodilatory mechanism in addition to ANP in 
the liver to counteract the portal hypertension of cirrhosis. It also follows that the increased CNP expression in liver tumour tissue may simply reflect increased stimulation from the high ANP concentrations, as there was no parallel increase in CNP receptor in tumour tissue.

The identification of these local hepatic natriuretic peptide systems is a very exciting one. The question still remains as to how significant are these findings. The presence of increased values of ANP and CNP transcripts together with their mRNAs coding for all three NPR types does not necessarily mean biological significance. Further data including evidence of increased cyclic GMP production together with evidence of blocking by specific antibodies will be required to answer this question. Furthermore, the question of whether ANP is coming from hepatoma cells or endothelial cells and whether they are being secreted into the blood will have to be clarified. For example, measuring the hormone levels in the hepatic veins draining the tumour versus the veins draining the hepatic parenchyma might help to resolve this. As so often in science, these interesting findings raise more questions than they answer. If we can be sure that these peptides have biological activity, then potentially, they could be used as tools to study the many pathophysiological changes associated with cirrhosis. The use of clearance receptor blockers could increase natriuretic peptide levels and augment their known effects. Likewise, if ANP is responsible for liver tumour growth, then the use of receptor blockers could hold promise in the treatment of liver tumours. However, how these goals could be achieved remains a challenge.

Department of Medicine,

FLORENCE WONG

The Toronto Hospital,

University of Toronto,

Toronto, Ontario, Canada

Correspondence to: $\mathrm{Dr}$ L Blendis, 9EN/220, The Toronto Hospital, 200 Elizabeth Street, Toronto M5G 2C4, Ontario, Canada.

1 de Bold AJ, Borenstein HB, Veress AT, Sonneberg H. A rapid and potent natriuretic response to intravenous injection of atrial myocardial extract in rats. Life Sci 1981; 28: 89-94.

2 Florkowski CM, Richards AM, Espiner EA, et al. Renal, endocrine and hemodynamic interactions of atrial and brain natriuretic peptides in normal man. Am $¥$ Physiol 1994; 266: R1244-50.

3 Morishita R, Gibbons GH, Pratt RE, et al. Autocrine and paracrine effects of atrial natriuretic peptide gene transfer on vascular smooth muscle and of atrial natriuretic peptide gene transfer on vascular smooth

4 Komatsu Y, Itoh H, Suga S-I, Ogawa Y, Hama N, Kishmoto I, et al. Regulation of endothelial production of C-type natriuretic peptide in coculture with vascular smooth muscle cells. Role of the vascular natriuretic peptide system in vascular growth inhibition. Circ Res 1996; 78: 606-14.

5 Komatsu Y, Nakao K, Itoh H, et al. Vascular natriuretic peptide. Lancet 1992; 340: 622 .

6 Gines P, Jimenez W, Arroyo V, et al. Atrial natriuretic factor in cirrhosis with ascites: plasma levels, cardiac release and splanchnic extraction. Hepatology 1988; 8: 636-42.

7 Wong F, Sniderman K, Liu P, Allidina Y, Sherman M, Blendis L. Transjugular intrahepatic portosystemic stent shunt: effects on hemodynamics and sodium homeostasis in cirrhosis and refractory ascites. Ann Intern Med 1995; 122: 816-22.

8 Nazario B, Hu RM, Pedram A, et al. Atrial and brain natriuretic peptides stimulate the production and secretion of C-type natriuretic peptide from bovine aortic endothelial cells. F Clin Invest 1995; 95: 1151-7. 